\begin{tabular}{|c|c|}
\hline hommes & $\begin{array}{l}\text { Hommes \& migrations } \\
\text { Revue française de référence sur les dynamiques }\end{array}$ \\
\hline & $\begin{array}{l}1316 \mid 2017 \\
\text { L'islam en Europe }\end{array}$ \\
\hline
\end{tabular}

\title{
Les musulmans à l'intérieur de la « Maison néerlandaise »
}

De l'importance de l'origine dans les politiques définissant l'appartenance nationale

\section{Jan Willem Duyvendak et Marieke Slootman}

Traducteur : Catherine Guilyardi

\section{(2) OpenEdition Journals}

\section{Édition électronique}

URL : http://journals.openedition.org/hommesmigrations/3784

DOI : 10.4000/hommesmigrations.3784

ISSN : 2262-3353

\section{Éditeur}

Musée national de l'histoire de l'immigration

\section{Édition imprimée}

Date de publication : 1 mars 2017

Pagination : 9-15

ISBN : 978-2-919040-37-7

ISSN : $1142-852 X$

\section{Référence électronique}

Jan Willem Duyvendak et Marieke Slootman, « Les musulmans à l'intérieur de la « Maison

néerlandaise » », Hommes \& migrations [En ligne], 1316 | 2017, mis en ligne le 01 mars 2020, consulté le 19 septembre 2020. URL : http://journals.openedition.org/hommesmigrations/3784 ; DOl : https:// doi.org/10.4000/hommesmigrations.3784 


\title{
LES MUSULMANS À L'INTÉRIEUR DE LA «MAISON NÉERLANDAISE 》 DE L'IMPORTANCE DE L'ORIGINE DANS LES POLITIQUES DÉFINISSANT L'APPARTENANCE NATIONALE
}

Par JAN WILLEM DUYVENDAK, professeur de sociologie, université d'Amsterdam et MARIEKE SLOOTMAN, docteure en anthropologie, université d'Amsterdam.

\begin{abstract}
La construction de l'autre en négatif de la définition de soi est au cœur de la relation biaisée qu'entretiennent les Pays-Bas avec leurs citoyens musulmans. Derrière son image d'Épinal d'un peuple ouvert et accueillant, la société néerlandaise impose les représentations d'une culture nationale auxquelles les immigrés et leurs descendants sont sommés de s'adapter. Les visions protectionnistes des libertés culturelles ou sexuelles, et nationalistes du territoire fonctionnent de concert dans cet imaginaire qui fantasme son homogénéité. L'identité néerlandaise se fabrique ainsi sur une double discrimination religieuse et ethnique dont ceux qu'elle vise ne parviennent pas à s'extraire.
\end{abstract}

Comme dans beaucoup d'autres pays, l'islam est de plus en plus présenté, depuis une vingtaine d'années, comme la principale limite et source de clivages dans la société néerlandaise. Les musulmans sont perçus comme une menace contre - ce qui est considéré comme - la culture néerlandaise. Les citoyens nés de parents turcs ou marocains sont dépeints comme étrangers parce que musulmans et porteurs d'une culture " traditionnelle » - donc incompatible avec les valeurs et la culture néerlandaises. Être Hollandais est de plus en plus souvent défini comme le fait d'être moderne, individualiste, progressiste (par exemple, tolérer l'homosexualité) et séculaire. Le caractère central de la religion dans la formulation de l'appartenance - ou non - à la nation contraste avec la situation dans certains pays, comme les États-Unis, où la religion, en tant que telle, n'est pas autant mise en cause. Cela a des conséquences sur la signification que revêt le fait d'être musulman, particulièrement dans le contexte politique néerlandais où, en matière d'appartenance nationale, « l'identification » à la nation ainsi que la « culture " prédominent. Pour reprendre les termes d'une députée qui s'adressait à des immigrés extracommunautaires 
et à leurs descendants aux Pays-Bas : " Les immigrés doivent clairement exprimer leur engagement visà-vis de leur nouveau "chez-eux" " (Tineke HuizingaHeringa au Parlement néerlandais en 2004¹). Non seulement les immigrés et leurs enfants doivent se sentir chez eux et s'identifier à leur pays de résidence, mais aussi intégrer ce qui leur est renvoyé comme étant « la » culture nationale.

\section{Le prétendu échec du « multiculturalisme »}

Le discours qui exige cet engagement viscéral découle de l'idée que «l'intégration » des immigrés a échoué à cause de la stratégie politique du multiculturalisme libéral ${ }^{2}$. Jusqu'à la seconde moitié des années 1980, les immigrés qui arrivaient des pays méditerranéens, en tant que main-d'œuvre non qualifiée, n'avaient pas - et n'étaient pas encouragés - à s'adapter à la culture et à l'identité néerlandaises. Les Pays-Bas avaient donc une image de tolérance. Tous les partis politiques, ou presque, affirment encore aujourd'hui, à tort, que l'idéal multiculturaliste n'exigeait pas des immigrés de s'assimiler, mais les encourageait, au contraire, à garder leurs « propres » identité, langue et culture. Cette politique de « maintien » des étrangers dans leur culture d'origine n'a rien à voir avec un idéal politique de multiculturalisme ${ }^{3}$. Elle s'est construite sur le présupposé, relayé par les immigrés euxmêmes comme par la société dans son ensemble, qu'ils retourneraient au Maroc ou en Turquie. Ils étaient donc toujours renvoyés à leur pays d'origine, même longtemps après leur immigration. Les politiques mises en place visaient à faciliter le retour envisagé ${ }^{4}$. C'est dans ce contexte que le maintien de leurs " propres » culture et langue était encouragé. Bien que de nombreux immigrés soient finalement restés aux Pays-Bas, le gouvernement a poursuivi cette politique, notamment avec les Marocains et les Turcs, jusqu'à la fin des années 1980. Pourtant, l'objectif avait changé, passant de l'aide au retour à une intégration socio-économique ${ }^{5}$.

Les différences socioculturelles des immigrés nétaient donc pas mises en avant pour leur valeur intrinsèque, comme le suppose le véritable « multiculturalisme ». Ce malentendu a été entretenu par de nombreux hommes politiques et chercheurs ${ }^{6}$. Au contraire, comme l'un d'entre nous l'a affirmé ailleurs, ces dernières décennies, ces différences ont généré de plus en plus de malaise ${ }^{7}$.

\section{Une culturalisation de la citoyenneté}

De nombreux immigrés et leurs descendants, natifs des Pays-Bas, se battent encore pour se sentir chez eux dans ce pays ${ }^{8}$. Selon les hommes

1. Voir Jan Willem Duyvendak, The Politics of Home. Belonging and Nostalgia in Western Europe and the United States, New York, Palgrave Macmillan, 2011, p. 100. 2. Ruut Koopmans, Paul Statham, Marco Giugni, Florence Passy, Contested Citizenship. Immigration and Cultural Diversity in Europe, Minneapolis, University of Minnesota Press, 2005 ; Paul Sniderman, Louk Hagendoorn, When Ways of Life Collide: Multiculturalism and Its Discontents in the Netherlands, Princeton, Princeton University Press. 2006. 3. Christophe Bertossi, Jan Willem Duyvendak, Martin Schain (dir.), « The problems with national models of integration: A Franco-Dutch comparison ", in Comparative European Politics, vol. 10, $n^{\circ}$ 3, 2012. 4. Peter Scholten, Framing Immigrant Integration: Dutch ResearchPolicy Dialogues in Comparative Perspective, Amsterdam, Amsterdam University Press, 2011. 5. Nadia Bouras, Het Land van Herkomst. Perspectieven op Verbondenheid met Marokko, 1960-2010, Hilversum, Uitgeverij Verloren, 2012 ; Marlou Schrover, « Pillarization, multiculturalism et cultural freezing. Dutch migration history and the enforcement of essentialist ideas ", in $B M G N$, vol. 125, $n^{\circ} 3-4$, 2010, pp. 329-354. 6. Voir Ruut Koopmans et al., Contested Citizenship..., op. cit.; Paul Sniderman, Louk Hagendoorn, op. cit. Pour une critique de cette interprétation multiculturaliste des politiques hollandaises, voir Jan Willem Duyvendak, Trees Pels, Rally Rijkschroeff, " A multicultural paradise? The cultural factor in dutch integration policy », in Jennifer L. Hochschild, John H. Mollenkopf (dir.), Bringing Outsiders In. Transatlantic Perspectives on Immigrant Political Incorporation, Ithaca, Cornell University Press, 2009, pp. 129-139; Jan Willem Duyvendak, Peter Scholten, « Deconstructing the dutch multicultural model: A frame perspective on dutch immigrant integration policymaking ", in Comparative European Politics, vol. 10, n 3, 2012, pp. 266-282 ; Jan Willem Duyvendak, Rogier van Reekum, Fatiha El-Hajjari, Christophe Bertossi, « Mysterious multiculturalism. The risks of using model-based indices for making meaningful comparisons ", in Comparative European Politics, vol. 11, n 5, 2013, pp. 599-620. 7. Evelien Tonkens, Menno Hurenkamp, Jan Willem Duyvendak, « Culturalization of Citizenship in the Netherlands », in Ariane C. d'Appollonia, Simon Reich (dir.), Managing Ethnic Diversity After 9/11. Integration, Security and Civil Liberties in Transatlantic Perspective, New Brunswick, Rutgers University Press, 2010, pp. 233-252 ; Menno Hurenkamp, Evelien Tonkens, Jan Willem Duyvendak, Crafting Citizenship. Negotiating Tensions in Modern Society, Basingstoke, Palgrave Macmillan, 2012. 8. Jan Willem Duyvendak, The Politics of Home, op. cit. 
politiques, la solution repose sur une plus grande cohésion sociale et une " citoyenneté néerlandaise partagée ». Mais cela pose la question de ce qu'ont ou devraient avoir en commun les différents groupes ethniques et religieux dans la nation, les villes ou les quartiers. Comment les Néerlandais peuvent-ils dessiner ensemble les contours de valeurs démocratiques communes et de ce qui relève du domaine public? La réponse hollandaise - due, en partie, à la réaction à l'essai Le drame multiculturel de Scheffer, en 2000, qui accuse les élites d'être trop " relativistes » et propose la création d'une identité nationale forte - fut de mettre la citoyenneté au premier plan dans l'appartenance nationale. Cette dernière ne repose pas, comme on pourrait s'y attendre, sur les éléments traditionnels de la citoyenneté : les devoirs formels et les droits judiciaires des membres de la communauté politique. Elle repose plutôt sur des éléments culturels dans le but de dessiner les contours d'une communauté culturellement homogène.

La «bonne citoyenneté » ne dépend pas, en premier lieu, du fait de travailler, de payer des impôts ou de voter, mais de pratiques culturelles " correctes ", de vêtements « acceptables " pour les femmes, de sentiment d'appartenance et de loyauté, de sécularisme et de l'affichage de "sentiments " appropriés et exprimés au bon moment ${ }^{9}$. Ce besoin de s'adapter à la culture nationale néerlandaise (non seulement aux lois de la nation, mais aux attentes culturelles non écrites) a été formulé explicitement dans une note d'orientation politique du ministère de l'Intégration: "Les fondements de la vie sociale aux Pays-Bas sont le résultat d'une histoire et de points d'ancrage que beaucoup de Néerlandais partagent et qui ne doivent pas disparaître. Il ne s'agit pas uniquement des valeurs qui fondent la Nation néerlandaise, mais des références qui ont évolué historiquement et culturellement, tels que la langue, certains monuments, des caractéristiques architecturales, des cou- tumes et comportements non-écrits qui se sont développés au cours de l'histoire ${ }^{10}$. "

Ce discours sur la culturalisation de la citoyenneté n'a pas seulement envahi l'arène politique, mais a imprimé sa marque sur les politiques actuelles. Des programmes d'intégration civique obligatoires sur les coutumes «néerlandaises » et l'histoire des Pays-Bas ont été, par exemple, mis en place pour les immigrés extracommunautaires (y compris ceux qui vivent depuis des décennies dans le pays). Pour être accepté comme un citoyen à part entière, on doit se sentir " chez soi » aux Pays-Bas, montrer que l'on connaît les traditions et pratique les coutumes «néerlandaises » et que l'on a intériorisé les mœurs "néerlandais ». C'est ce que nous appelons la " culturalisation de la citoyenneté11 ". Elle requiert une relation affective des migrants avec leur nouvelle nation. Ce discours sur l'appartenance nationale, basé sur des éléments culturels et émotionnels, semble très exigeant et plutôt excluant.

\section{Définir l'identité néerlandaise progressiste}

Comment cette identité nationale - cette " hollandité (Dutchness) »- se définit-elle ? Est-elle plutôt ouverte et inclusive envers les immigrés ? Ou les immigrés sont-ils paradoxalement enjoints d'intégrer une identité qui ne leur sera jamais reconnue? Nous allons montrer qu'il s'agit plutôt de la dernière assertion. L'identité et la nation néerlandaises reposent sur une conception essentialiste et sans fondement historique de ce qui n'est pas néerlandais. L'appartenance est définie par rapport à un Autre culturel, en particulier l'immigré musulman ${ }^{12}$. Katherine Verdery parle du «mythe de l'homogénéité » fabriqué par les hommes d'État pour créer de la cohésion nationale. Comme elle l'explique, le sentiment de similarité implique l'exclusion et la différenciation, parce 
que «nous » ne peut être défini qu'en opposition à "eux»; et «la culture » est un des domaines privilégiés où cela est possible ${ }^{13}$.

Pratiquement tous les partis politiques, y compris les populistes de droite, définissent les valeurs «modernes » et «progressistes » - en particulier dans le domaine de la religion, du genre et de la sexualité - comme des caractéristiques fondamentales des Néerlandais. Le sécularisme, l'égalité des genres et l'acceptation de l'homosexualité servent de repères idéologiques pour tester l'entrée dans la " modernité " des immigrés, unique condition pour avoir l'autorisation d'appartenir à la société néerlandaise.

Les travaux récents sur les nouveaux clivages politiques en Europe de l'Ouest ont souvent ignoré le rôle central joué par la rhétorique du progrès concernant la sexualité et le genre. La montée du populisme est donc analysée, à tort, comme un changement
Pour être accepté comme

un citoyen à part entière,

on doit se sentir «chez soi » aux Pays-Bas, montrer que

l'on connaît les traditions

et pratique les coutumes « néerlandaises » et que l’on

a intériorisé les mœurs «néerlandais». linéaire vers le conservatisme. Cette incompréhension est due à l'amalgame fait entre les idées progressistes et des points de vue favorables aux immigrés. Notre analyse montre que les populistes défendent une culture nationale néerlandaise progressiste sur les questions de société, en même temps qu'un programme virulent contre les immigrés.

Quand les politologues Kriesi et al. constatent que "le libéralisme culturel des plus éduqués a décliné de manière considérable, reflétant probablement [...] la crispation générale du discours néerlandais contre les immigrés ${ }^{14}$ ", ils passent à côté d'un point central qui implique que ce discours va de pair avec une rhétorique sur l'égalité des genres et l'émancipation sexuelle. La tolérance vis-à-vis d'un groupe tel que la communauté LGBT n'implique pas la tolérance vis-à-vis d'autres différences, comme l'ethnicité ou la religion.

\section{Les Néerlandais marocains et turcs : ces « Autres » immigrés et musulmans}

Dans ce discours, qui définit l'identité néerlandaise comme progressiste et séculière, les immigrés originaires du Maroc et, dans une moindre mesure, de Turquie sont présentés comme ces "Autres » culturels qui permettent de dessiner les contours de l'identité néerlandaise. Cette mise en exergue des Hollandais marocains repose sur leurs situations socio-économique et socioculturelle. Les problèmes sociaux, tels que leur surreprésentation dans des zones à forte criminalité, avec des troubles à l'ordre public et un échec scolaire importants, ont contribué à créer cette image de la culture marocaine comme étant "arriérée ». Pour bien comprendre pourquoi les Marocains sont supposés " ne pas être intégrés » aux Pays-Bas, il convient de préciser que la culture marocaine a été récemment remplacée par «l'islam ».

Les musulmans les plus nombreux sont les Marocains et les Turcs. Ce sont aussi les groupes ethniques minoritaires les plus importants. Les estimations les plus récentes sur l'appartenance religieuse datent de 2008 : il y a 296000 Marocains d'origine et 285000 Turcs d'origine pour 825000 musulmans $^{15}$. Dans le discours national, l'ensemble des Marocains et des Turcs sont confondus avec la catégorie "musulmans ". Aujourd'hui, les deuxième et troisième générations représentent respectivement $2,2 \%$ et 2,4 \% de la population néerlandaise (375 000 et 396000 sur un total de 16 millions, selon le Bureau central des statistiques néerlandaises ${ }^{16}$ ).

13. Katherine Verdery, «Ethnicity, nationalism and state-making. Ethnic groups and boundaries: past and future », in Hans Vermeulen, Cora Govers (dir.), The Anthropology of Ethnicity: Beyond Ethnic Groups and Boundaries, Amsterdam, Het Spinhuis, 2000, pp. 45-46. 14. Hanspeter Kriesi, Edgar Grande, Martin Dolezal, Marc Helbling, Dominic Höglinger, Swen Hutter, Bruno Wüest, Political Conflict in Western Europe, Cambridge, Cambridge University Press, 2012, p. 171. 15. Estimations du Bureau central des statistiques néerlandaises. Voir Statistics Netherlands, Moslim in Nederland, La Haye, Centraal Bureau voor de Statistiek (CBS) [Bureau central des statistiques néerlandais], 2012, p. 44. 16. Statistics Netherlands, Jaarrapport Integratie 2014, La Haye, Centraal Bureau voor de Statistiek (CBS) [Bureau central des statistiques néerlandais], 2014 
La proportion est beaucoup plus importante dans les grandes villes. Par exemple, $28 \%$ des enfants de 10 ans à Amsterdam sont originaires du Maroc ou de la Turquie ${ }^{17}$. Dans certains quartiers d'Amsterdam et de Rotterdam, les Néerlandais marocains et turcs représentent ensemble de 40 à $50 \%$ de la population. Ce sont les groupes ethniques les plus importants, particulièrement parmi les jeunes, et établis depuis le plus longtemps dans ces quartiers, même par rapport aux Néerlandais ${ }^{18}$.

La première génération de Marocains est arrivée aux Pays-Bas à la fin des années 1960 et dans les années 1970. C'était de jeunes hommes, des «travailleurs invités » comme main-d'œuvre non qualifiée ${ }^{19}$. Nombre d'entre eux venaient de zones rurales et n'avaient pas un niveau d'études élevé. La plupart étaient musulmans. Quand il devint clair qu'ils resteraient, leur famille les a rejoints. La plupart des familles observaient des règles et coutumes traditionnelles et une bonne partie de la deuxième génération - comparée aux Néerlandais plutôt séculiers et athées - pratiquent leur religion et/ou se définissent comme «musulmans ».

Alors que la plupart des migrants marocains ont un faible niveau d'éducation et sont restés dans les catégories socio-économiques les plus basses, la deuxième génération a connu une promotion sociale importante. Depuis les années 1990, la proportion des jeunes d'origine marocaine et turque parmi les nouveaux étudiants a grimpé de $20 \%$ à plus de $40 \%{ }^{20}$. Certes, un groupe important de jeunes est resté sur le bord de la route ${ }^{21}$ : malgré son augmentation constante, le niveau moyen d'éducation de la deuxième génération est toujours plus bas que celui des Néerlandais d'origine ${ }^{22}$.

L'islam est de plus en plus associé, et dans de nombreux pays, à une image irréconciliable - de façon inhérente - avec les valeurs « progressistes » de l'Occident $^{23}$. L'islam, en tant que religion, a remplacé l'ethnicité marocaine comme marqueur de l'autre culture, une entité culturellement distincte (marocaine ou musulmane) de l'identité néerlandaise. Ethnicité et religion sont utilisées pour mettre en avant ce qui est irréconciliable avec ce qui est vu comme la culture néerlandaise. Ces barrières culturelles, basées sur le religieux, excluent davantage que celles basées sur l'ethnicité, dans la mesure où elles résonnent avec le discours global, et très essentialisant, de la « guerre des civilisations ».

L'immigration des musulmans est perçue comme une menace pour la stabilité de l'ordre moral néerlandais séculaire et libéral. Des Dans ce discours, qui définit l'identité néerlandaise comme progressiste et séculière, les immigrés originaires du Maroc et, dans une moindre mesure, de Turquie sont présentés comme ces « Autres» culturels qui permettent de dessiner les contours de l'identité néerlandaise. protectionnistes culturels se sont érigés comme protecteurs des libertés culturelles et sexuelles des Néerlandais contre les dangers supposés des immigrés musulmans ${ }^{24}$. Bien que les acteurs politiques n'expriment pas tous avec la même intensité ce discours d'exclusion, le populiste Geert Wilders a réussi à attirer l'attention des médias et un soutien électoral significatif avec son discours très anti-immigrés et antimusulmans. Il a, par exemple, diffusé un autocollant montrant le drapeau saoudien recouvert du slogan : "L'islam est un mensonge, Mahomet est un criminel, le Coran est un poison. » Au printemps 2014, il a déclaré qu'il voulait « moins de Marocains » et, quand la salle entière a repris en chœur son slogan, il a répondu : "Nous allons nous en occuper. "Son parti est arrivé en tête des élections européennes en 2014.

17. Ibid., p. 67. 18. Maurice Crul, Jens Schneider, Frans Lelie (dir.), The European Second Generation Compared. Does the Integration Context Matter?, Amsterdam, Amsterdam University Press, 2012.19. Hans Vermeulen, Rinus Penninx (dir.), Immigrant Integration. The Dutch Case, Amsterdam, Het Spinhuis, 2000.20. Statistics Netherlands, Jaarrapport Integratie 2012, La Haye, Centraal Bureau voor de Statistiek (CBS) [Bureau central des statistiques néerlandais], 2012 p. 85. 21. Maurice Crul, Jeroen Doomernik, "The turkish and moroccan second generation in the Netherlands: Divergent trends between and polarization within the two groups ", in The International Migration Review, vol. 37, $\mathrm{n}^{\circ}$ 4, 2003, pp. 1039-1064. 22. Statistics Netherlands, Jaarrapport Integratie 2012, op. cit. 23. Justus Uitermark, Paul Mepschen, Jan Willem Duyvendak, "Populism, sexual politics and the exclusion of muslims in the Netherlands ", in John R. Bowen, Christophe Bertossi, Jan Willem Duyvendak, Mona Krook (dir.), European States and their Muslim Citizens, Cambridge, Cambridge University Press, 2014, pp. 235-255. 24. Peter Van der Veer, " Pim Fortuyn, Theo van Gogh et the politics of tolerance in the Netherlands », in Public Culture, vol. 18, $n^{\circ} 1,2006$, pp. 111-124. 


\section{Le nativisme dans l'identité néerlandaise : de l'importance de l'origine}

L'adaptation culturelle est-elle donc la clé pour être accepté (au sein de la nation) ? Les politiciens néerlandais considéreront-ils un jour que les immigrés musulmans peuvent s'assimiler ? Pour répondre à ces questions, nous devons considérer un autre niveau d'exclusion dans le discours néerlandais dominant : le nativisme. Il est ancré dans l'idée que ceux dont les familles sont installées sur le territoire néerlandais depuis des siècles ont plus de légitimité à parler de "notre » culture et de "notre » identité, qu'ils ont plus de droits et, finalement, appartiennent

Le discours néerlandais basé sur la culture, dans leque

I'islam a fini par être dépeint comme diffèrent de façon inhérente de «la » culture nationale, est commun à de nombreux pays. Cependant, la proportion dans laquelle l'islam est perçu comme étant en opposition totale avec la culture nationale des Pays-Bas semble inégalée (à l'exception du Danemark peut être) "plus » à la nation ${ }^{25}$. La profondeur de ce nativisme néerlandais s'illustre par l'emploi répandu et constant du terme "allochtone " (qui veut dire littéralement : " pas de ce sol $^{26}$ ») pour désigner les immigrés «nonOccidentaux » et leurs enfants, et parfois leurs petits-enfants, par opposition aux « autochtonen " (" de ce sol »). Ce qui amène des enfants nés aux Pays-

Bas, dont une grande majorité est de nationalité néerlandaise, à être considérés de façon persistante comme des étrangers.

Ce nativisme rend le processus d'appartenance nationale encore plus excluant. Alors que les exigences basées sur le sentiment d'appartenance et la « culture " supposent que l'adaptation de la première et deuxième génération est possible, qu'ils seront donc acceptés en tant que citoyens à part entière, l'exigence nativiste implique que les immigrés et leurs descendants directs n'appartiendront jamais à la nation, en tout cas pas autant que les Néerlandais de "souche » qui sont pleinement et supposés seuls véritables citoyens de ce pays. Les minorités, particulièrement les musulmans, sont donc considérées avec suspicion, quelles que soient leurs pratiques et identifications culturelles. La position nativiste, présente dans le discours populiste, dépeint non seulement les migrants musulmans comme étrangers (non-natifs) à la culture néerlandaise, mais aussi comme non assimilables. Le fossé entre immigrés et natifs du pays est donc par définition infranchissable. Certes, les immigrés peuvent, peut-être et avec le temps, devenir de plus en plus "natifs ", mais ils ne deviendront jamais de « vrais » natifs.

Ce discours rend pleinement légitime l'aliénation de ceux qui ont une origine immigrée et place les pratiques d'exclusion au-dessus de toute critique. Les politiques de tous bords envoient donc un message paradoxal aux immigrés, les assignant à s'assimiler totalement à une culture à laquelle il est admis qu'ils ne pourront jamais s'assimiler.

Le discours néerlandais basé sur la culture, dans lequel l'islam a fini par être dépeint comme diffèrent de façon inhérente de "la » culture nationale, est commun à de nombreux pays. Cependant, la proportion dans laquelle l'islam est perçu comme étant en opposition totale avec la culture nationale des Pays-Bas semble inégalée (à l'exception du Danemark peut être).

\section{Impact du discours sur la citoyenneté des musulmans}

Quel impact ce discours sur la citoyenneté aux Pays Bas, qui se concentre de plus en plus sur « l'étrangeté » culturelle et religieuse des musulmans, a-t-il sur le fait d'être musulman pour la seconde génération de Marocains-Hollandais ? Et que peut-on dire du discours néerlandais sur lequel se basent nos résultats empiriques? Des données quantitatives et qualitatives nous permettent de conclure 
que l'identification à l'islam est relativement forte parmi cette deuxième génération. Cela s'explique, pour certains, par la valeur intrinsèque de la religion et la religiosité personnelle. Être musulman procure une source d'inspiration et des règles de vie, ainsi que le sentiment d'appartenir à une communauté mondiale. De plus, pour la deuxième génération de Marocains néerlandais, être musulman est dans la droite ligne de leur éducation et constitue une manière de garder le lien avec leurs parents. Cependant, leur choix d'être musulman ne peut être étranger au contexte politique d'exclusion. L'importance sociétale de la religion, en tant que marqueur social, augmente l'importance de leur identité musulmane en tant qu'individu et augmente leur orientation vers l'islam. Dans ce sens, nous pouvons dire qu'être musulman est en partie une attitude réactive.

Mais quelle est la nature de cette " réaction " ? Est-elle de l'ordre de la tradition ou résolument moderne ? La deuxième génération ne singe pas l'expérience religieuse de leurs parents. Être musulman évolue avec le temps et la signification de l'islam, pour ces jeunes, est loin d'être évidente. Ils fabriquent leur propre version dé-culturalisée de la religion, dans laquelle ils nourrissent un lien avec leurs parents tout en négociant l'espace dans lequel ils participent, à leur façon, à la société hollandaise. Ils utilisent l'islam pour transcender l'opposition entre Marocains et Néerlandais, pour créer leurs propres espace et identité tournés vers la société, tout en étant enracinés dans leur milieu musulman et marocain. Pour eux, l'islam n'est pas nécessairement en opposition avec l'appartenance à une société "moderne » comme celle des PaysBas. Une perspective qui met en question le discours culturaliste néerlandais.
Nous pouvons conclure que le débat public influence la façon d'être musulman aux PaysBas, comme beaucoup d'autres facteurs. L'accent mis continuellement par la société sur " l'islam » et une "identité musulmane ", qui serait identique quel que soit l'individu, rend très visible la part religieuse de l'identité de ces jeunes. Ils se lancent dans une recherche consciente et personnelle du sens de l'islam, alors même qu'ils souffrent de la place de l'islam devenu, dans le discours politique dominant, un outil pour définir, par opposition, l'identité néerlandaise.

Pour de nombreux musul-
L’islam, même « dé-ethnicisé

»- c'est-à-dire un islam qui l'être Marocain - et de plus en plus moderne sur plusieurs aspects, n'aide pas celui qui s'en revendique à être accepté au sein de la nation néerlandaise. sépare l'être musulman de mans aux Pays-Bas, où être

Hollandais est si profondément défini comme " séculier » et "progressiste ", cette définition oppositionnelle reste problématique. L'islam, même " dé-ethnicisé " - c'est-à-dire un islam qui sépare l'être musulman de l'être Marocain - et de plus en plus moderne sur plusieurs aspects, n'aide pas celui qui s'en revendique à être accepté au sein de la nation néerlandaise. Tant que cette pratique religieuse évolutive ne sera pas reconnue et continuera à être vue comme traditionnelle, comme tournant le dos à la société hollandaise, tant que l'accent sera mis de façon excessive sur le rôle supposé crucial de la religion et de l'islam présenté comme irrémédiablement " arriéré » et incompatible avec le fait d'être un Néerlandais «natif », tant que ce discours ne changera pas, les efforts des jeunes musulmans pour être considérés comme des citoyens à part entière resteront complexes. Traduit de l'anglais par Catherine Guilyardi. 